\title{
Estudo de resinas acrílicas modificadas por diferentes tamanhos de partícula para aplicação na área odontológica
}

\author{
Study of acrylic resins modified by different \\ particles sizes for application in \\ odontology area.
}

\author{
${ }^{1}$ Universidade Sociedade Educacional de Santa Catarina - UNISOCIESC, CEP: 89206-001, Joinville, Santa Catarina, \\ Brasil. \\ e-mail: tiago.vicente@ hotmail.com.br; palova@ sociesc.org.br
}

\begin{abstract}
RESUMO
Uma vez que partículas inorgânicas exercem papel fundamental nas propriedades de uma resina composta na odontologia, buscou-se avaliar a influência da variação do tamanho médio das partículas em suas propriedades mecânicas. Foram avaliados nestas resinas odontológicas a resistência à flexão, resistência à compressão, microdurezaVickers e tensão de contração. Para evitar variações indesejadas, a consistência destas resinas não variou entre as amostras (Consistência Squeeze $=75 \mathrm{~N}$ ). Além da consistência, outras variáveis de uma resina composta (composição da matriz orgânica, iniciadores, inibidores) tiveram suas quantidades fixadas, buscando não haver outro tipo de influência no estudo. Foram utilizados nove tipos de resinas nesse trabalho, com tamanho médio de partícula variando entre $0,4 \mu \mathrm{m}$ e 3,0 $\mu \mathrm{m}$, havendo três resinas compostas por tamanho único de partícula $(0,4 \mu \mathrm{m}, 1,0 \mu \mathrm{m}$ e $3,0 \mu \mathrm{m})$ e seis resinas compostas por misturas das cargas citadas anteriormente. Os resultados obtidos foram compilados e os dados submetidos à análise estatística (ANOVA - Teste de Comparações Múltiplas de Tukey com significância de 5\%). Os resultados demonstraram que o efeito de sinergia recorrente da mistura de cargas acarretou em uma elevação nos valores de resistência à compressão e resistência à flexão, se comparados a resinas com cargas de tamanho único. Também se pode constatar que não há uma única faixa de tamanho médio de partículas com resultados superiores às demais em todas as propriedades. Buscando um balanço entre as propriedades analisadas, as amostras com tamanho médio de partículas de 2,02 $\mu \mathrm{m}$ e 2,14 $\mu \mathrm{m}$, ambas compostas por misturas de cargas (não carga única), apresentaram melhores resultados. Conforme análise estatística de Tukey, ambas foram posicionadas como pertencentes aos 2 agrupamentos que indicam propriedades superiores em todos os ensaios realizados.
\end{abstract}

Palavras-chave: resina acrílica, propriedades mecânicas, tamanho de partícula.

\section{ABSTRACT}

With the increase in population life expectancy, the search for products with greater durability increases. This leads to search for more and more resistant materials, including in the dental field. Once inorganic particles play a fundamental role in the properties of a composite resin, we searched to evaluate the influence different particles size in its mechanical properties. In these dental resins, we evaluated flexural strength, compressive strength, microhardness Vickers and contraction stress. To avoid unwanted variations, the consistency of these resins did not differ between samples (Consistency Squeeze $=75 \mathrm{~N}$ ). Besides consistency, other variables of a composite resin (organic matrix composition, initiators, inhibitors) had their amounts fixed, seeking no other influence on the study. Nine types of resins were used in this study, with an average particle size ranging between $0.4 \mu \mathrm{m}$ and $3.0 \mu \mathrm{m}$, there are three resins composed of single particles $(0.4 \mu \mathrm{m}, 1.0 \mu \mathrm{m}$ e 3.0 $\mu \mathrm{m})$ and six resins composed by mixing particles mentioned above. The results were compiled and the data submitted to statistical analysis (ANOVA - Multiple Comparisons Tukey test with 5\% significance). The results showed that the synergistic effect of the mixture of particles reflected better properties in compressive strength and flexural strength test as compared to resins with single-size particles. Is possible to conclude too that there is not a single average size range of particles with results superior to other resins in all properties. 
However, looking for a balance between these properties, samples with an average particle size of $2.02 \mu \mathrm{m}$ and $2.14 \mu \mathrm{m}$ both composed of mixtures of particles (no single particles) showed the best results. As showed by Tukey's statistical analysis, both resins were placed as belonging to the 2 groups that indicate superior properties in all tests.

Keywords: acrylic resins, mechanical properties, particle size.

\section{INTRODUÇÃO}

Com o avanço da ciência e da tecnologia, o mundo passa por diversas mudanças, vive em constante evolução. Paralelo a isso, conforme BATISTA[1], a expectativa de vida da população cresce e a exigência por produtos que acompanham esse duradouro ciclo de vida, vem sendo foco de estudo.

Na área odontológica não é diferente, há uma busca por materiais que não apenas preencham as cavidades oriundas de cáries, mas também mantenham a eficiência da capacidade mastigatória e agreguem boa estética. A liga metálica, denominada amálgama dentária assumiu esse papel no passado, porém, apenas na parte de propriedades mecânicas, já que possuía péssima estética e alta expansão na cavidade dentária. Na década de 60, a síntese do monômero Bisfenol A Glicedil Dimetacrilato (BisGMA), criado por Bowen, resultou na grande evolução dos materiais dentários. Possibilitando a criação de resinas compostas que conciliam ótima estética e boas propriedades mecânicas, buscando características similares a estrutura dentária, prolongando o ciclo de vida do material e mantendo a saúde bucal do paciente [2]. Basicamente essa resina é formada por uma matriz resinosa orgânica (constituída prioritariamente por BisGMA e monômeros diluentes), carga inorgânica e um agente de união, no qual a matriz orgânica é formada pelos monômeros e é unida às partículas de carga por meio do agente de ligação [3,4].

Reforçando a importância dessa evolução citada por SILVA et al. [2], ANUSAVICE et al.[5] afirmam que resinas compostas utilizadas para restauração na área odontológica, fraturam após poucos anos de uso devido à força de mastigação exercida pelo paciente ao final do ciclo mastigatório. A tensão necessária para que ocorra essa fratura tende a ser menor no decorrer dos anos devido à propagação lenta de defeitos minúsculos que se tornam uma microtrinca através do processo de fadiga cíclica. Assim, a adição de cargas à matriz orgânica serve para aumentar a rigidez, resistência mecânica e resistência ao desgaste; e também reduzir a expansão térmica, sorção de água e tensão de contração, consequentemente, minimizando o problema previamente citado.

Logo, a importância desse trabalho se reflete em melhorar as propriedades da resina acrílica odontológica, atributos esses que buscam reproduzir como o material irá se comportar quando utilizado em áreas de alto estresse funcional no ambiente oral, e com esse ganho de qualidade, assegurar maior durabilidade em sua aplicação clínica [6]. Entre essas propriedades, a tensão de contração (uma das propriedades analisada nesse estudo) das resinas compostas acrílicas na área odontológica é foco de estudo. De acordo com QVIST et al. [7], a alta tensão de contração pode acarretar na ocorrência de falhas na interface entre a resina composta e o dente, resultando em infiltração marginal. Essa situação pode aumentar a probabilidade do aparecimento de cáries secundárias, degradação marginal, sensibilidade pós-operatória e em casos de maior gravidade, podem acarretar fratura coesiva na estrutura dentária, assim, gerando sérios danos à saúde bucal do paciente.

A minimização desses problemas é importante para a área de saúde odontológica, a qual pode trazer grande benefício ao tratamento clínico, aumentando a eficiência e longevidade das restaurações e elevando a qualidade de vida dos pacientes tratados.

O que diferencia esse trabalho de vários outros é a possibilidade da síntese dessas resinas em laboratório, minimizando variáveis encontradas em estudos onde são utilizadas resinas comerciais, como a variação no percentual de iniciadores, a variedade de monômeros utilizados na matriz orgânica, as diferentes composições das partículas inorgânicas, a diferença de consistência, etc. Sendo assim, a mistura de cargas e seu teor final na resina, serão as únicas variáveis no estudo, todos os demais parâmetros serão padronizados.

Com isso, esse trabalho delimita seu estudo na influência do tamanho de partículas e na influência da sinergia entre elas, mantendo a consistência semelhante entre as amostras, na busca de melhores propriedades que atestam maior durabilidade em ciclo oral.

\section{MATERIAIS E MÉTODOS}

A Figura 1 apresenta as etapas do trabalho, sendo o mesmo dividido em seis etapas. A etapa 1 representa a dissolução de fotoiniciadores, co-iniciadores (diacetona, amina terciária aromática) e inibidores de polimerização, a etapa 2 representa a homogeneização da matriz orgânica. Na etapa 3 é demonstrada a preparação das cargas (utilizado cargas da marca Schott Dental Glass - Classe GM27884, compostas de 55\% Dióxido de 
Silício, 25\% Óxido de Bário, 10\% Óxido de Boro e 10\% Óxido de Alumínio, tratadas por agente de união silano, com tamanho de partícula de $0,4 \mu \mathrm{m}, 1,0 \mu \mathrm{m}$ e 3,0 $\mathrm{mm}$ ) e a incorporação dessas na matriz orgânica. Já a etapa 4 exemplifica a análise e ajuste de consistência e para finalizar as etapas 5 e 6 representam respectivamente a análise de tensão de contração e ensaios mecânicos.

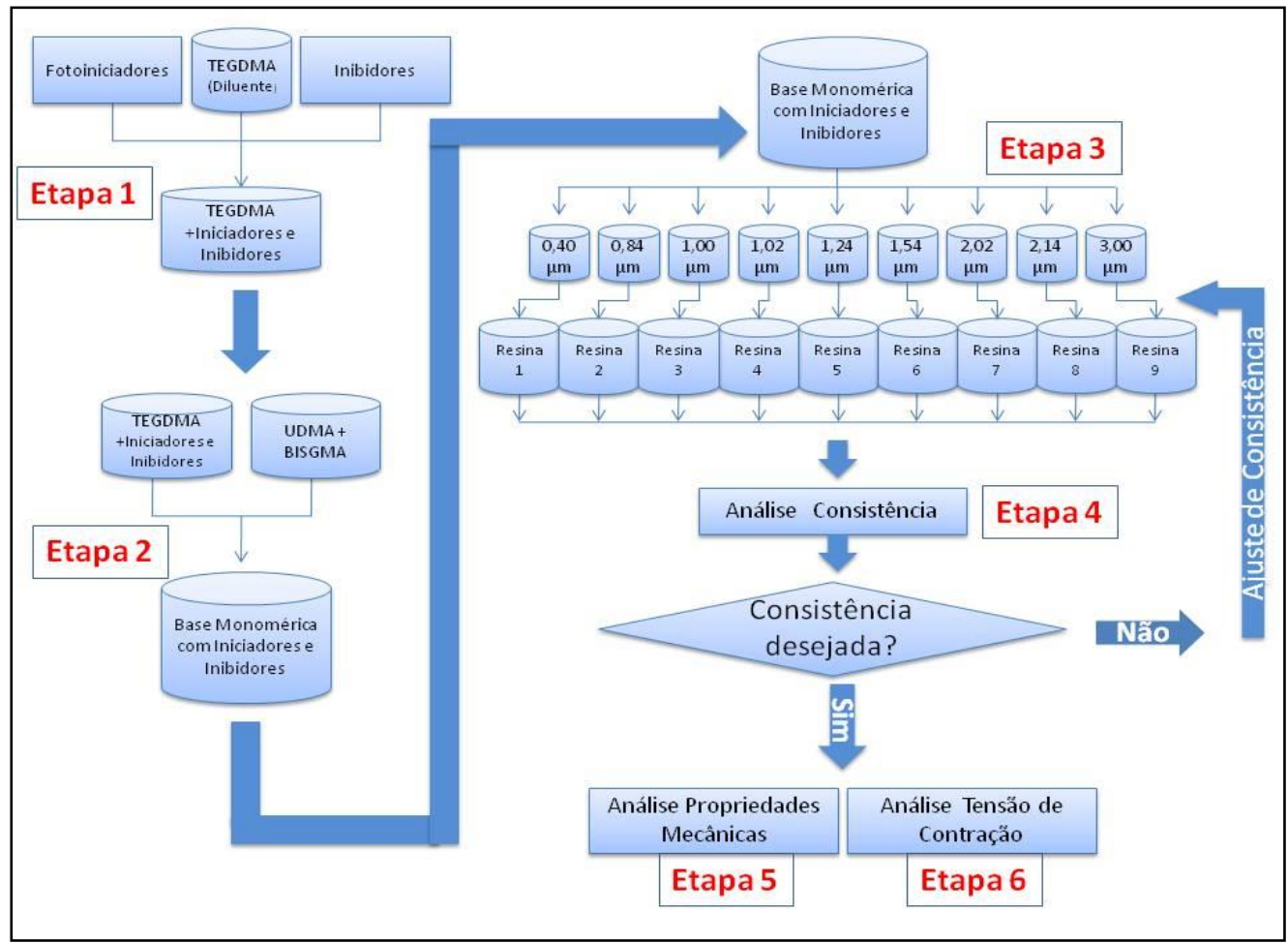

Figura 1: Etapas de desenvolvimento do trabalho.

\subsection{Dissolução de Iniciadores, Co-iniciadores e Inibidores}

Primeiramente, os iniciadores, co-iniciadores e inibidores de polimerização foram adicionados simultaneamente ao monômero TEGDMA (Triethylene Glycol Dimethacrylate - Esstech Inc), que tem baixa viscosidade e facilita a dissolução dos mesmos. A quantidade de iniciadores, co-iniciadores e inibidores, foi mantida constante.

Essa solução foi levada ao equipamento Ultrassom Inox Ultra Cleaner, até total dissolução de sólidos. Para que não houvesse perda de eficiência do sistema a temperatura foi controlada, até $40^{\circ} \mathrm{C}$, a medição de temperatura foi realizada por termômetro digital MT-330.

\subsection{Homogeneização da Matriz Orgânica}

A matriz orgânica utilizada na síntese das resinas compostas deste trabalho foi integrada exclusivamente pelos monômeros tradicionalmente utilizados nas resinas dentárias comerciais [5], BisGMA (Esstech Inc.), UDMA (Urethane Dimethacrylate - Esstech Inc.), e TEGDMA (Triethylene Glycol Dimethacrylate - Esstech Inc.).

A homogeneização da matriz orgânica foi realizada no aparelho SpeedMixer DAC 150.1 FVZ-K CM 47, com três ciclos de 30 segundos, rotação de $3500 \mathrm{rpm}$, intercalados por um espaçamento de três minutos entre cada ciclo, visando manter a matriz orgânica a temperaturas aceitáveis.

\subsection{Preparação das Cargas}

Foram preparadas nove amostras de resinas compostas, utilizando matriz orgânica padrão (citada no item 2.2) e com variação no tamanho médio de partículas, sendo seis formadas por misturas de cargas e três formadas por partículas de tamanho único. As partículas de vidro foram estabelecidas buscando parametrizar o mesmo fornecedor (Schott Dental Glass) e a mesma classe (GM27884), essa que conforme citado, representa a composição química das partículas. Já o tamanho de partícula foi definido conforme disponibilizado pelo forne- 
cedor, desde que atendesse aos parâmetros acima. Sendo de conhecimento que o fornecedor dispõe de partículas da classe GM27884 nos tamanhos de 0,4 $\mu \mathrm{m}, 0,7 \mu \mathrm{m}, 1,0 \mu \mathrm{m}, 2,0 \mu \mathrm{m}$ e 3,0 $\mu \mathrm{m}$, logo, ficou definida a utilização das partículas de tamanho $0,4 \mu \mathrm{m}, 1,0 \mu \mathrm{m}$ e $3,0 \mu \mathrm{m}$, evitando a utilização de partículas de diâmetros próximos. Essa distribuição estabelecida é apresentada na Tabela 1, levando em conta que o tamanho médio demonstrado foi calculado através da média ponderada entre os teores de carga da resina composta.

Tabela 1: Distribuição percentual em peso, do tamanho de partícula inorgânica em cada formulação de resina composta utilizada nesse estudo.

\begin{tabular}{l|l|l|l|l}
\hline \multirow{2}{*}{ FORMULAÇÃo } & \multicolumn{2}{l|}{\begin{tabular}{l}
\multicolumn{2}{l}{ QUANTIDADE POR TAMANHO } \\
DE PARTÍCULA (\%)
\end{tabular}} & TAMANHO MÉDIO $(\boldsymbol{\mu m})$ \\
\cline { 2 - 5 } & $0,4 \mu \mathrm{m}$ & $1,0 \mu \mathrm{m}$ & $3,0 \mu \mathrm{m}$ & \\
\hline 01 & 100 & 0 & 0 & 0,40 \\
02 & 60 & 30 & 10 & 0,84 \\
03 & 0 & 100 & 0 & 1,00 \\
04 & 30 & 60 & 10 & 1,02 \\
05 & 60 & 10 & 30 & 1,24 \\
06 & 10 & 60 & 30 & 1,54 \\
07 & 30 & 10 & 60 & 2,02 \\
08 & 10 & 30 & 60 & 2,14 \\
09 & 0 & 0 & 100 & 3,00 \\
\hline
\end{tabular}

Obs.: O percentual carga/monômero utilizado será definido conforme a consistência de cada formulação, esse percentual sofrerá variação e será ajustado buscando padronizar a consistência entre as mesmas, com adição de matriz orgânica ou de sua blenda de carga correspondente.

As cargas foram homogeneizadas em aparelho SpeedMixer DAC 150.1 FVZ-K CM 47, com rotação de 3000 rpm em três ciclos de 15 segundos, espaçados por cinco minutos de repouso.

\subsection{Incorporação das Cargas Inorgânicas na Matriz Orgânica}

Para obtenção dos compósitos, as cargas foram incorporadas na matriz utilizando aparelho SpeedMixer DAC 150.1 FVZ-K CM 47, com rotação de $3000 \mathrm{rpm}$ em 10 ciclos de 25 segundos, intercalados por um espaçamento mínimo de cinco minutos entre cada ciclo, mantendo a temperatura abaixo dos $40^{\circ} \mathrm{C}$, com acompanhamento via termômetro digital MT-330.

Após completa homogeneização, com auxílio de uma bomba de alto vácuo Edwards, modelo E2MB e acessório do aparelho SpeedMixer DAC 150.1 FVZ-K CM 47, foi aplicado vácuo durante 60 segundos (Figura 2), seguido por ciclo rotacional de 20 segundos a $3000 \mathrm{rpm}$. Essas duas etapas foram repetidas quatro vezes para retirada de gases inerentes ao processo, os quais podem reduzir as propriedades mecânicas do material [8].

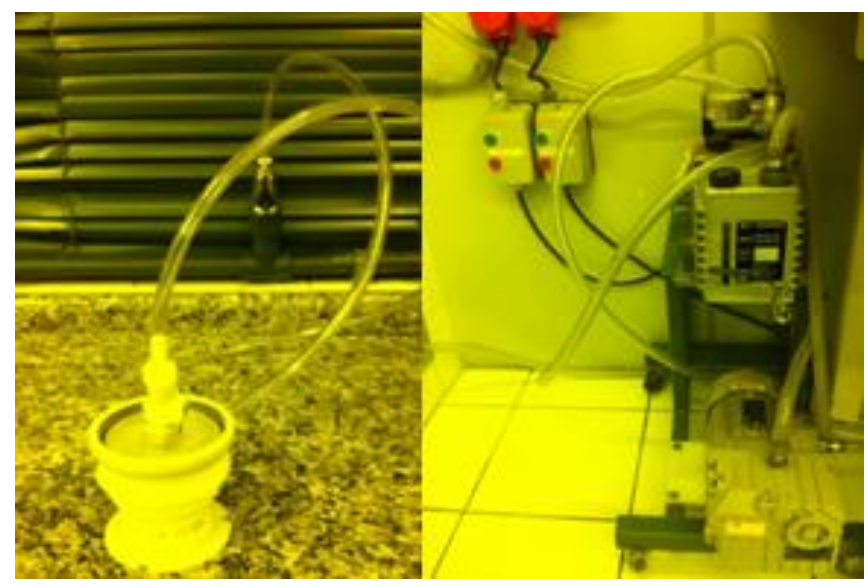


Figura 2: Bomba de alto vácuo e acessório para retirada de gases inerentes ao processo.

Após a retirada de gases e completa homogeneização, foi realizada a análise preliminar de consistência através de método de espatulação manual, sequencialmente, foram realizados os ajustes necessários nos teores de matriz orgânica e partículas inorgânicas até que atingida a consistência uniforme entre as resinas em análise (a consistência foi mantida uniforme buscando que todas as amostras possam atender a mesma necessidade do profissional de odontologia).

\subsection{Análise e Ajuste de Consistência}

A análise da consistência final da resina composta foi realizada via ensaio de consistência pelo método Squeeze, junto à máquina universal de ensaios EMIC DL-3000. A máquina foi parametrizada com célula de $500 \mathrm{~N}$, velocidade de ensaio de $5 \mathrm{~mm} / \mathrm{min}$ e tempo de ensaio igual a 2 minutos. As resinas compostas foram preparadas com massa de 20 gramas, inseridas em molde de poliacetal com formato cilíndrico (figura 3) e analisadas em triplicata. As amostras foram mantidas a $21^{\circ} \mathrm{C}$ no momento imediatamente antes da realização do ensaio.

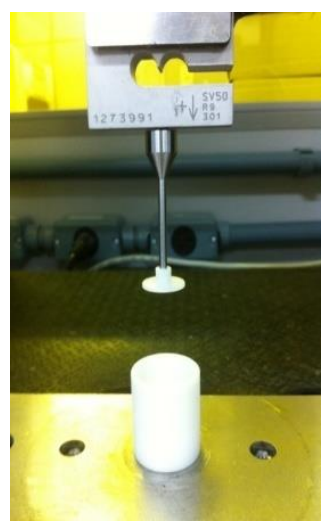

Figura 3: Máquina de ensaios universal e resina composta inserida em molde cilíndrico de poliacetal.

As resinas compostas apresentaram variação de consistência entre si, logo, essa diferença foi corrigida adicionando maior teor de monômeros/respectiva carga (conforme necessidade de ajuste) e repetido o procedimento de homogeneização da resina. Sendo assim, as resinas foram reanalisadas e reajustadas, afim de não mais apresentar diferença considerável de consistência entre as amostras. Esse estudo aceitou uma variação de consistência pelo método Squeeze de $\pm 10 \mathrm{~N}$, como desvio não considerável de consistência. Agregando esse desvio aceitável, ao valor conhecido de consistência Squeeze de uma resina acrílica odontológica para restauração comercial (Filtek Z250 - 3M/ESPE - $75 \mathrm{~N}$ ), esse estudo manteve a consistência de suas amostras entre 65 e $85 \mathrm{~N}$.

\subsection{Ensaios Mecânicos}

Para análise das propriedades mecânicas das resinas compostas, foram realizados quatro tipos de ensaios: tensão de contração, resistência à compressão, resistência à flexão em três pontos e microdureza vickers.

O ensaio de tensão de contração foi realizado na máquina universal de ensaios EMIC DL - 3000 com célula de carga de $500 \mathrm{~N}$. Foram utilizadas duas bases de aço de formato cilíndrico, com diâmetro de $6 \mathrm{~mm}$, posicionadas paralelamente, sendo uma conectada ao braço móvel e outra ao braço fixo da máquina. A superfície de ambas as bases foi tratada por adesivo odontológico Ambar (FGM - Dentscare), polimerizado por 20 segundos com fotopolimerizador Emitter C Schuster, de luz LED, com o intuito de gerar adesividade entre a superfície metálica e a resina odontológica. Então, as resinas compostas foram introduzidas entre as bases metálicas, que se encontram separadas por um espaçamento de 1,5 $\mathrm{mm}$.

As bases permaneceram fixas durante o ensaio, e as forças geradas durante a polimerização foram registradas através de uma curva tensão x tempo. A polimerização foi realizada utilizando fotopolimerizador Emitter C Schuster, de luz LED, com potência medida previamente $\left(1250 \mathrm{~mW} / \mathrm{cm}^{2}\right)$ com radiômetro modelo BTM 2000, e comprimento de onda entre 420 e $480 \mathrm{~nm}$. A polimerização foi efetuada durante 60 segundos em 3 pontos equidistantes (20 segundos cada ponto) e o tempo total da análise foi de 10 minutos para cada 
amostra, com valor fixado de massa igual a $0,11 \mathrm{~g}$, sendo analisado em triplicata.

O ensaio de resistência à compressão foi realizado na máquina universal de ensaios EMIC DL-3000, com célula de carga de $20 \mathrm{KN}$ e velocidade de ensaio igual a $0,5 \mathrm{~mm} / \mathrm{min}$, com a análise de 6 amostras. Cada corpo de prova foi obtido a partir de um molde em aço inoxidável com diâmetro de $3 \mathrm{~mm}$ e altura de $6 \mathrm{~mm}$, fotopolimerizados com LED luz azul (Emiter C Schuster) com densidade de potência de $1250 \mathrm{~mW} / \mathrm{cm}^{2}$.

Para analisar a microdureza, foi utilizado o método Vickers com o Microdurômetro MV 2000 A Digital e realizado análise em quintuplicata, com medição única no centro de cada um dos 5 corpos de prova. Cada corpo de prova foi obtido a partir de um molde de $5 \mathrm{~mm}$ de diâmetro e $0,82 \mathrm{~mm}$ de espessura e a polimerização da amostra foi realizada utilizando fotopolimerizador Emitter C Schuster, de luz LED, com potência medida previamente $\left(1250 \mathrm{~mW} / \mathrm{cm}^{2}\right)$, durante 30 segundos em cada lado da amostra.

O ensaio de Flexão em três pontos (norma ISO 4049:2009) foi realizado na máquina universal de ensaios EMIC DL-3000 com célula de carga de $500 \mathrm{~N}$, velocidade de deslocamento do cutelo de 1,00 $\mathrm{mm} / \mathrm{min}$ com auxílio de um deflectômetro. Cada corpo de prova foi obtido a partir de um molde em aço inoxidável com dimensões $25 \times 2 \times 2 \mathrm{~mm}$, sendo essa análise em quintuplicata. As resinas compostas foram fotopolimerizadas para ensaio com LED de luz azul (Emiter C Schuster) com densidade de potência de $1250 \mathrm{~mW} / \mathrm{cm}^{2}$.

Para realização das análises citadas acima (exceção tensão de contração), inicialmente, os corpos de prova foram mantidos por 24 horas com água deionizada em estufa a $37^{\circ} \mathrm{C}$, sendo a análise realizada somente após esse período. Procedimento esse recomendado pela norma ISO 4049:2009.

\subsection{Análise Estatística}

Os dados foram submetidos à ANOVA - Teste de Comparações Múltiplas de Tukey, o qual foi aplicado a 5\% de probabilidade ( $\mathrm{p}<0,05$ = nível de significância), assim as médias foram agrupadas, sendo que dentro desses agrupamentos, letras diferentes indicam diferença significativa e letras iguais indicam diferença estatisticamente não considerável. Foi adotado por esse estudo que as amostras com melhores resultados foram alocadas como agrupamento A, sendo sucedida pelos agrupamentos B, C, D e E.

\section{RESULTADOS E DISCUSSÃO}

Primeiramente todas as amostras foram ajustadas a fim de manter consistência uniforme entre elas, a qual foi verificada através de análise de consistência Squeeze.

Devido as suas características reológicas, cada amostra necessitou de um teor de carga diferente para atingir a mesma consistência. Essa variação no teor de carga é detalhada na Figura 4.

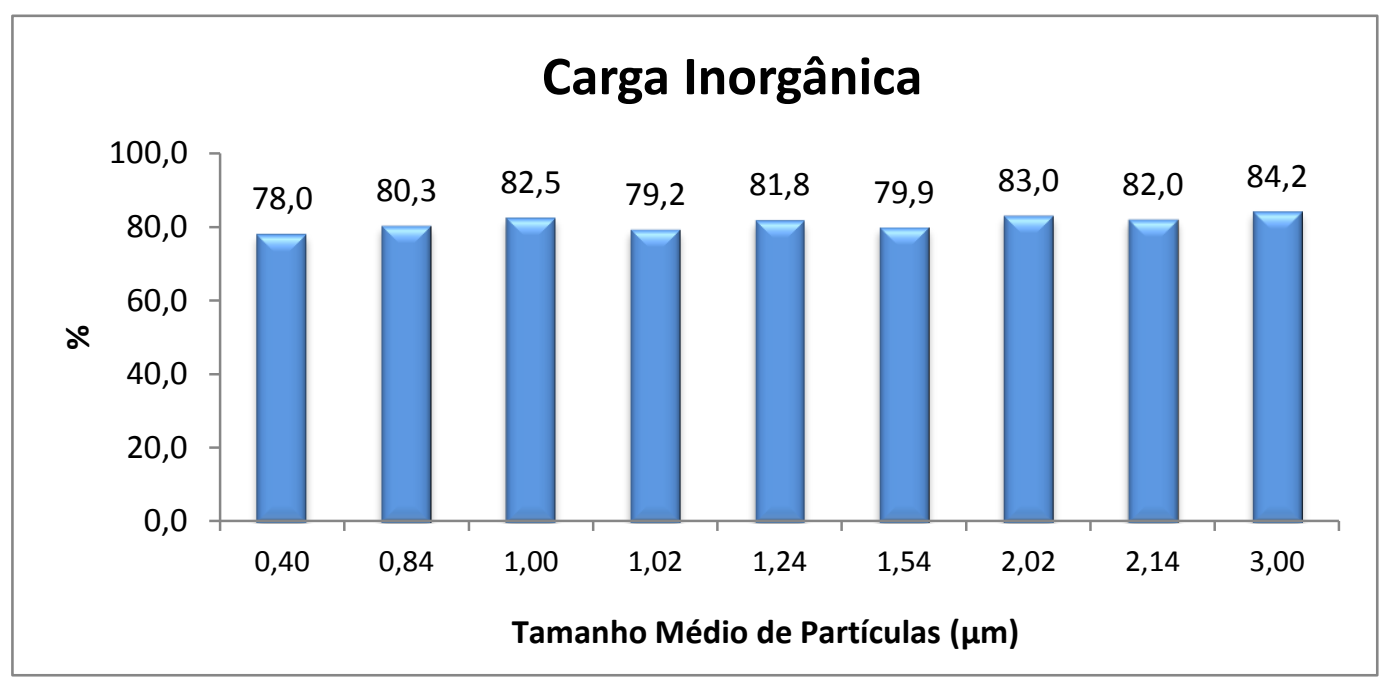

Figura 4: Percentual final de carga em cada resina composta em estudo.

As amostras de 1 a 9 estão dispostas em ordem crescente, levando em conta o tamanho médio de partícula conforme visualizado na Figura 3, assim a amostra 1 apresenta menor tamanho médio de partícula e a amostra 9, maior.

Conforme resultados apresentados na Figura 3, a resina com menor tamanho médio de partículas $(0,4$ 
$\mu \mathrm{m})$ foi a amostra que necessitou de menor teor de carga (78\%) para atingir a consistência desejada, por outro lado, a resina com maior tamanho médio de partículas $(3,0 \mu \mathrm{m})$, necessitou maior teor de carga $(84,2 \%)$ para atingir a mesma consistência. Essa análise corrobora com o conceito de ANUSAVICE et al. [5], que afirmam que quanto menor o diâmetro da partícula, maior a sua área superficial e consequentemente maior o número de ligações possíveis entre monômero e agente de ligação, constatando diminuição da fluidez e aumento da viscosidade. Esse efeito também foi observado por CHEN et al. [9], onde em um de seus experimentos, utilizando partículas de tamanho reduzido e consequentemente com maior área superficial, evidenciou redução no percentual máximo de enchimento da resina por partículas inorgânicas.

Porém, em uma análise individual, nem todos os experimentos se comportaram exatamente conforme esse conceito. As amostras entre 2 e 8 oscilaram quanto ao percentual de carga em cada resina, não estabelecendo um padrão, esse resultado pode ser explicado devido a pequena variação existente quanto ao tamanho médio de partícula entre amostras. A diferença entre o menor tamanho médio de partícula $(0,4 \mu \mathrm{m})$ e o maior $(3,0 \mu \mathrm{m})$ é pequena, levando em consideração que de acordo com FONSECA [10], ainda hoje são utilizadas partículas desde 0,005 a 100,0 $\mu \mathrm{m}$, logo, o conceito de ANUSAVICE et al. [5] e CHEN et al. [9] é evidenciado mais claramente ao analisar os extremos (Amostras 1 e 9).

\subsection{Análise de Propriedades}

Os resultados dos ensaios de microdurezaVickers, tensão de contração, resistência à compressão e resistência à flexão serão apresentados na Tabela 2. Os mesmos foram submetidos à análise estatística pelo teste de comparações múltiplas de Tukey ( $\mathrm{p}<0,05$ = nível de significância), conforme demonstrado na mesma tabela.

Tabela 2: Média aritmética, desvio padrão e análise estatística de Tukey para ensaios de microdurezaVickers, tensão de contração, resistência à compressão e resistência à flexão.

\begin{tabular}{|c|c|c|c|c|c|}
\hline \multirow[t]{2}{*}{ AMOSTRA } & \multirow{2}{*}{$\begin{array}{l}\text { TAMANHO } \\
\text { MÉDIO DE } \\
\text { PARTÍCULA } \\
(\mu \mathrm{M})\end{array}$} & \multicolumn{4}{|l|}{ ENSAIOS } \\
\hline & & $\begin{array}{l}\text { Microdureza- } \\
\text { Vickers }\end{array}$ & $\begin{array}{l}\text { Tensão de } \\
\text { Contração }\end{array}$ & $\begin{array}{l}\text { Resistência à } \\
\text { Compressão }\end{array}$ & Resistência à Flexão \\
\hline 1 & 0,40 & $64,17^{c} \pm(2,8)$ & $2,20^{\mathrm{e}} \pm(0,02)$ & $344,3^{a b} \pm(21,8)$ & $123,9^{b} \pm(7,9)$ \\
\hline 2 & 0,84 & $68,89^{\mathrm{bc}} \pm(3,5)$ & $2,01^{\mathrm{cd}} \pm(0,05)$ & $362,0^{\mathrm{a}} \pm(32,8)$ & $149,6^{\mathrm{a}} \pm(8,5)$ \\
\hline 3 & 1,00 & $71,23^{\mathrm{abc}} \pm(2,6)$ & $2,06^{\mathrm{de}} \pm(0,03)$ & $321,1^{\mathrm{b}} \pm(16,1)$ & $131,5^{\mathrm{ab}} \pm(5,5)$ \\
\hline 4 & 1,02 & $72,58^{a b c} \pm(6,5)$ & $2,02^{\mathrm{d}} \pm(0,09)$ & $338,5^{\mathrm{ab}} \pm(17,3)$ & $145,6^{\mathrm{ab}} \pm(6,7)$ \\
\hline 5 & 1,24 & $79,69^{\mathrm{a}} \pm(1,2)$ & $1,99^{\mathrm{cd}} \pm(0,02)$ & $345,4^{\mathrm{ab}} \pm(20,1)$ & $146,8^{\mathrm{a}} \pm(8,6)$ \\
\hline 6 & 1,54 & $72,30^{\mathrm{abc}} \pm(1,7)$ & $1,94^{\mathrm{bcd}} \pm(0,08)$ & $302,5^{\mathrm{c}} \pm(4,2)$ & $143,8^{a b} \pm(12,7)$ \\
\hline 7 & 2,02 & $75,91^{\mathrm{ab}_{ \pm}} \pm(4,1)$ & $1,85^{b c} \pm(0,01)$ & $318,6^{b c} \pm(13,9)$ & $140,7^{\mathrm{ab}_{ \pm}}(15,6)$ \\
\hline 8 & 2,14 & $75,38^{a b} \pm(1,4)$ & $1,80^{\mathrm{b}} \pm(0,09)$ & $315,8^{b c} \pm(15,1)$ & $138,7^{\mathrm{ab}} \pm(14,9)$ \\
\hline 9 & 3,00 & $76,49^{\mathrm{ab}} \pm(2,4)$ & $1,60^{\mathrm{a}} \pm(0,05)$ & $266,7^{\mathrm{d}} \pm(6,6)$ & $135,5^{\mathrm{ab}} \pm(12,1)$ \\
\hline
\end{tabular}

Letras diferentes indicam resultados com diferença significante entre amostras (Tukey $-\mathrm{p}<0,05$ ).

\subsubsection{Microdureza Vickers}

O ensaio de microdurezaVickers pode ser considerado uma análise de superfície, que tem o objetivo de mensurar a resistência de superfícies sólidas a endentação, visto que segundo RODRIGUES [11], materiais com baixa dureza de superfícies são suscetíveis à rugosidade. De acordo com CRAIG [4], essa rugosidade pode comprometer a resistência à fadiga do material, diminuindo o seu ciclo de vida em ambiente oral. Logo, valores maiores nesse ensaio resultam em melhores propriedades.

Sendo de conhecimento que o grupo A pela classificação de Tukey, representa maiores médias aritméticas e o grupo $\mathrm{C}$ menores, estatisticamente é demonstrado que o agrupamento A ficou concentrado nas amostras com tamanho médio de partículas maiores e o agrupamento $\mathrm{C}$ deslocado para as menores partículas (tabela 2).

Partindo dessa análise, observa-se que as amostras contendo maior quantidade de partículas menores apresentaram resultados inferiores de MicrodurezaVickers, se comparado as com partículas maiores.

Esse resultado pode ser proveniente do pressuposto que resinas compostas com partículas menores são constituídas de menor teor de cargas inorgânicas, logo, apresentando maior teor de matriz orgânica, assim, oferecendo menor resistência à endentação, corroborando com MONTENEGRO et al. [12], BEUN et al. [13] e KIM et al [14], que evidenciaram que resinas com menor teor de partículas inorgânicas apresentaram resultados relativamente menores. 


\subsubsection{Tensão de Contração}

Já o ensaio de Tensão de Contração tem o intuito de mensurar a contração da resina composta durante seu processo de polimerização, pois quanto menor a contração, menor o risco de ocorrer infiltração marginal [5] e maior o ciclo de vida da mesma em ambiente oral.

Para os resultados de Tensão de Contração, a análise estatística de Tukey apresenta maior diversidade de grupos, se comparado ao ensaio de microdureza (Tabela 2), demonstrando que essa propriedade apresentou variações mais significativas entre as amostras desse trabalho. Assim, se observou que essa propriedade tem maior sensibilidade quanto à mudança no tamanho de partículas na resina.

Os ensaios apresentaram típicas curvas de tensão x tempo, características para ensaios em resinas compostas odontológicas fotoativadas, onde [5], inicialmente ocorre maior contração e ao longo do tempo, após a conversão de boa parte dos monômeros, tende a estabilizar.

A Tabela 2 também demonstra que amostras com tamanho de partículas menores foram agrupadas próximas ao grupo E o qual atesta maior tensão de contração, e amostras com partículas maiores estão alocadas próximas ao grupo $\mathrm{A}$, o qual atesta menores tensões.

A diferença da média aritmética dos resultados de tensão de contração entre as amostras com menor tamanho médio de partícula $(0,40 \mu \mathrm{m})$ e maior $(3,00 \mu \mathrm{m})$ apresentam variação de $27 \%$, onde se pode observar que resinas com maior teor de partículas menores apresentam maior tensão de contração. Esse fato pode estar relacionado ao teor de partículas inorgânicas totais presentes na resina, visto que os grandes responsáveis pela tensão de contração são os monômeros [2,15], e as amostras com diâmetro médio de partícula menor apresentam maior percentual de monômero na resina, logo, se espera maior tensão de contração.

Corroborando com essa análise, AW e NICHOLLS [16] e MUNKSGAARD et al.[17], verificaram em seus experimentos que quanto menor a porcentagem de carga, maior a contração, pois conforme AW e NICHOLLS [16], as cargas inorgânicas são sólidas e não contraem, entretanto, a matriz orgânica é um fluido que contrai para virar sólido.

\subsubsection{Resistência à Compressão}

O ensaio de Resistência à Compressão busca reproduzir em laboratório as forças que ocorrem com alta frequência em materiais odontológicos durante o ciclo mastigatório e essas que influem diretamente na longevidade do compósito.

Os resultados de resistência à compressão conforme apresentado na análise estatística de Tukey (Tabela 2), apresentaram dispersão significativa, onde o agrupamento A representa resultado superior e o agrupamento D, inferior. De posse desses dados, se pode observar que as resinas compostas com maior concentração de partículas menores apresentaram tendência em resultados posicionados nos agrupamentos superiores (A e B), já as resinas com maior concentração de partículas maiores apresentaram deslocamento para os agrupamentos inferiores ( $\mathrm{C}$ e D). Essa tendência de resinas compostas formadas por partículas menores apresentarem melhores propriedades mecânicas também foi observado por TANIMOTO et al.[18] e MIYASAKA [19], onde TANIMOTO et al.[18] afirmam que em resinas compostas, a fratura inicial ocorre normalmente na interface matriz orgânica/partículas inorgânicas. Logo, ele evidenciou através de análise tridimensional por elementos finitos que partículas grandes tendem a gerar maior tensão na superfície, já resinas compostas por partículas menores, tendem a gerar maior energia superficial, pois quanto menores as partículas, maior a área superficial. Assim, essas características resultam em melhores propriedades de resistência à flexão e compressão para resinas constituídas de partículas menores.

Outro fator a ser observado, é quanto as amostras com maiores concentrações de cargas grandes tenderem a gerar maiores espaços sem preenchimento por partículas inorgânicas, os quais em grande parte são responsáveis por gerarem menor resistência nos materiais [5]. A Figura 5 exemplifica a aplicação de forças de compressão sobre diferentes distribuições de partículas, onde se pode avaliar que quanto menor a partícula, menor o espaço preenchido exclusivamente por matriz orgânica, porém, com a aplicação de uma mistura de cargas adequada, esses espaços podem ser reduzidos ainda mais. 


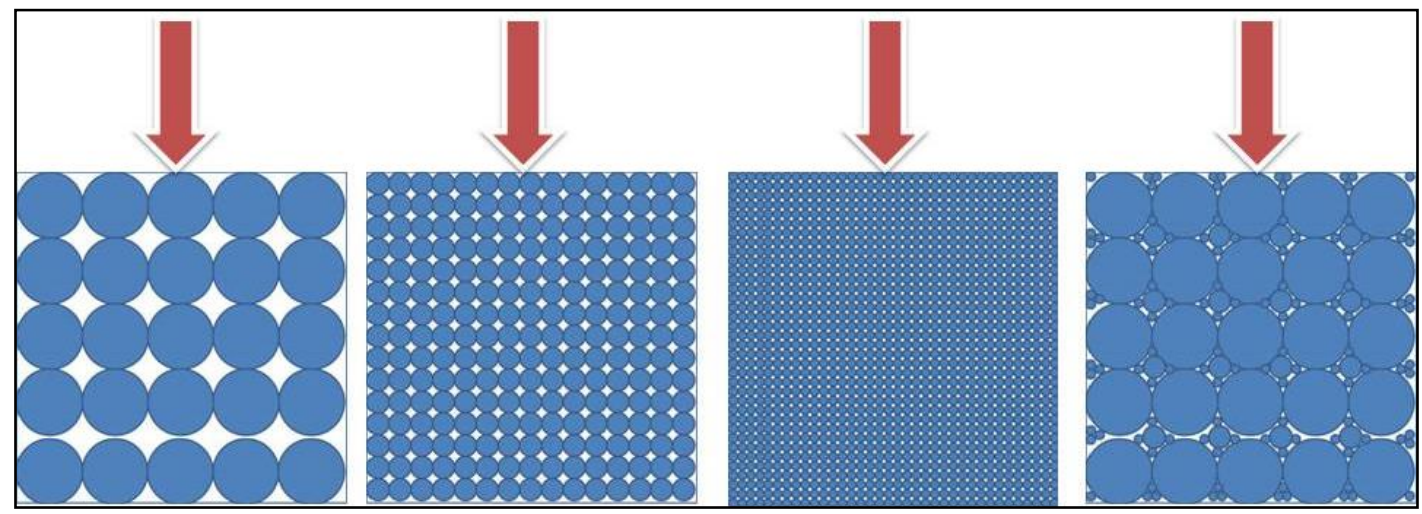

Figura 5: Diferentes tamanhos de partículas submetidas a forças de compressão.

Outra análise a ser considerada, parte do conceito [5] que uma distribuição de partículas com diferentes tamanhos pode levar a melhores propriedades mecânicas, devido ao menor percentual de pontos frágeis e de acordo com PUCKETT et al.[20], maior eficiência no empacotamento dos compósitos. Ainda citam que a busca por essa sinergia, trouxe ao mercado as resinas híbridas, micro-híbridas e nano-híbridas, com o intuito de maximizar o empacotamento e minimizar o tamanho de partícula, levando a ganhos em propriedades mecânicas e polimento.

Logo, sendo de conhecimento que as amostras $1(0,4 \mu \mathrm{m}), 3(1,0 \mu \mathrm{m})$ e $9(3,0 \mu \mathrm{m})$ desse presente estudo são compostas por partículas de tamanho único, enquanto as demais apresentam blendas variadas, se pode evidenciar que as blendas se sobressaem sobre as amostras de carga única quanto a resistência à compressão, corroborando com o estudo de Miyasaka [19], onde o autor destaca a importância da mistura de diversos tamanhos de partículas na propriedade de resistência à compressão.

Analisando as resinas constituídas de carga única com resinas de tamanho médio semelhantes constituídas de blendas, percebe-se que todas as amostras de carga única apresentam resultados não condizentes com o padrão observado anteriormente, onde resinas com menor tamanho médio de partículas apresentaram tendência a melhores propriedades mecânicas.

A amostra $1(0,40 \mu \mathrm{m}$ - carga única) apresentada na Tabela 2 foi classificada estatisticamente como pertencente ao grupo A e B, sendo que a amostra $2(0,84 \mu \mathrm{m}-$ mistura de cargas) apresentando mais que o dobro do seu tamanho médio, foi classificada como exclusivamente pertencente ao grupo A. Esse fato acontece de igual maneira na amostra $3(1,00 \mu \mathrm{m}$ - carga única) (Tabela 2), ela apresenta classificação exclusivamente B, enquanto a amostra anterior (2) apresenta classificação exclusivamente A, como já esperado. Porém, as amostras posteriores (4 e 5) foram classificadas como estatisticamente pertencentes aos grupos A e B, considerando-as assim com propriedades superiores a amostra 3. Para completar, a amostra $9(3,00 \mu \mathrm{m}-$ carga única) (Tabela 2), além de apresentar maior tamanho médio de partículas, apresenta carga única, em consequência desses fatores apresentou resultados aquém das demais resinas, sendo classificada como única representante do agrupamento D, o qual atesta menores propriedades de resistência à compressão.

\subsubsection{Resistência à Flexão}

Com o mesmo intuito do ensaio de resistência à compressão, porém analisando características diferentes, o ensaio de resistência à flexão em 3 pontos busca reproduzir em laboratório as forças que ocorrem no ciclo mastigatório e essas que influem diretamente na longevidade do compósito.

Analisando os dados estatísticos obtidos pelo método de Tukey, se observa que todas as amostras foram agrupadas em dois grandes grupos A e B (Tabela 2). Logo, pode-se dizer que a variação de carga nesse estudo apresentou pouca influência na variação da resistência à flexão. Porém, avaliando as médias aritméticas demonstradas igualmente na Tabela 2, pode-se aplicar, assim como na resistência à compressão, o conceito de efeito sinérgico proposto por Anusavice et al. [5] e corroborado por Miyasaka [19] e Puckett et al.[20], pois as amostras 1, 3 e 9 (cargas únicas), apresentaram os 3 piores resultados quanto a resistência à flexão, conforme detalhado na Tabela 3. 
Tabela 3: Comparação de médias aritméticas das leituras do Ensaio de Resistência à Flexão, ordenadas por ordem decrescente de propriedades.

\begin{tabular}{l|l|l|l}
\hline $\begin{array}{l}\text { RESISTÊNCIA À } \\
\text { FLEXÃO (MPA) }\end{array}$ & $\begin{array}{l}\text { BLENDA/CARGA } \\
\text { ÚNICA }\end{array}$ & $\begin{array}{l}\text { TAMANHO MÉDIO } \\
\text { DE PARTÍCULA }(\boldsymbol{\mu M})\end{array}$ & AMOSTRA \\
\hline 149,6 & Blenda & $\mathbf{0 , 8 4}$ & 2 \\
146,8 & Blenda & $\mathbf{1 , 2 4}$ & 5 \\
145,6 & Blenda & $\mathbf{1 , 0 2}$ & 4 \\
143,8 & Blenda & $\mathbf{1 , 5 4}$ & 6 \\
140,7 & Blenda & $\mathbf{2 , 0 2}$ & 7 \\
138,7 & Blenda & $\mathbf{2 , 1 4}$ & 8 \\
135,5 & Carga Única & 3,00 & 9 \\
131,5 & Carga Única & 1,00 & 3 \\
124,0 & Carga Única & 0,40 & 1 \\
\hline
\end{tabular}

Analisando aritmeticamente, pode-se observar que mesmo estatisticamente sendo pouco significativa, existe influência tanto do tamanho médio de partículas quanto no fator mistura de cargas/carga única. Pois excluindo as amostras com carga única (1, 3 e 9), a média aritmética demonstra tendência a apresentar melhores resultados, quanto menor o diâmetro médio de partículas conforme enfatizado (negrito) na Tabela 3 e corroborando com TANIMOTO et al. [18] que em seu estudo concluiu que quanto maior o tamanho de partícula, menor a resistência à flexão. Essas pequenas influências devem ser consideradas, pois a variação no tamanho de partículas realizadas nesse estudo pode ser considerada pequena, logo, com uma variação maior, possivelmente a diferença entre as misturas de cargas/carga única e o tamanho médio de partículas, resultaria em resultados com maior influência na resistência à flexão.

\section{CONCLUSÃO}

Tendo em vista os aspectos analisados, verificou-se através do ensaio de microdureza Vickers que superficialmente as resinas com maior percentual de cargas maiores apresentaram melhores propriedades, havendo uma diferença de $17 \%$ entre as amostras com cargas maiores e menores.

Através do ensaio de tensão de contração evidenciou-se que resinas com maior teor de cargas maiores apresentam menor tensão, ressaltando que estatisticamente essa propriedade foi a que sofreu maior influência com a mudança no tamanho de partículas e aritmeticamente apresentou maior diferença, sendo essa de $27 \%$ entre a amostra com maior concentração de partículas pequenas e a de menor concentração.

Quanto à análise de resistência à compressão, nota-se que blendas com partículas menores apresentam melhores resultados, se comparadas às resinas com concentração elevada de partículas maiores.

Na análise de Resistência à Flexão, observou-se que apesar dessa propriedade apresentar pequena ligação com o tamanho de partículas, houve uma leve tendência a partículas menores apresentarem resultados melhores.

Entretanto, a resistência à compressão e a resistência à flexão demonstram que o sinergismo entre tamanhos de partículas diferentes está diretamente ligado a melhores propriedades no interior da resina, pois resinas formadas com blendas de cargas se sobressaíram em todas as análises de compressão e flexão se comparado a resinas com carga única.

Diante dos resultados obtidos e de conhecimento da bibliografia disponível é possível afirmar que cada propriedade é influenciada de uma determinado maneira, não havendo uma determinada blenda de cargas que se sobressaia em todos os ensaios em estudo.

Por fim, partindo dessa ideia, pode-se assumir que as amostras 7 e 8, com tamanho médio de partículas de $2,02 \mu \mathrm{m}$ e $2,14 \mu \mathrm{m}$, respectivamente, ambas compostas por misturas de tamanhos variados de cargas, apresentaram estatisticamente um balanço melhor de resultados, pois ambas foram classificadas em todas as análises realizadas nesse estudo, como pertencentes aos dois agrupamentos estatísticos que representavam melhores resultados (Tukey A e B). Esse fato comprova que o sinergismo entre as cargas tem influência positiva quanto às propriedades mecânicas das resinas compostas. Outro fator observado, é que misturas de cargas com maior diâmetro médio de partícula apresentam um balanço melhor de propriedades, se comparado a mistura de cargas com tamanho médio de partícula inferior. 


\section{BIBLIOGRAFIA}

[1] BATISTA, V. CORREIO BRASILIENSE,

http://www.correiobraziliense.com.br/app/noticia/economia/2015/05/03/internas_economia,481695/avancotecnologico-e-mudancas-sociais-multiplicam-desafios-no-mercado.shtml, .Acessado em outubro de 2015.

[2] SILVA, J.M.F., et al., "Resinas compostas: estágio atual e perspectivas", Revista Odonto, v. 16, n. 32, pp. 98-104, Jul.-Dez. 2008.

[3] NOORT, R.V., Introdução aos materiais dentários, 3ed., Rio de Janeiro, Elsevier, 2010.

[4] CRAIG, R.G., Restorative dental materials, 10 ed., St. Louis, Mosby-Year Book Inc., 1997.

[5] ANUSAVICE, K.J., SHEN, C., RAWLS, H.R., Phillips materiais dentários, 12 ed., Rio de Janeiro, Elsevier, 2013.

[6] AGUIAR, F.H.B., BRACEIRO, A.T., AMBROSANO, G.M., et al., "Hardness and diametral tensile strength of a hybrid composite resin polymerized with different modes and immersed in ethanol or distilled water media", Dental Materials, v. 21, n. 12, pp. 1098-1103, Dez. 2005.

[7] QVIST, J., QVIST, V., MJÖR I.A., "Placement and longevity of amalgam restorations in Denmark", Acta Odontologica Scandinavica, v. 48, n. 5, pp. 305-311, Out. 1990.

[8] CHAIN, M.C., "Unidades fotoativadoras de luz visível - fotopolimerizdores", In: Baratieri, I.N. (ed), Estética: restaurações adesivas diretas em dentes anteriores, cap, 5, São Paulo, Livraria Santos, pp. 117-133, 1995.

[9] CHEN, M. H., CHEN, C.R., HSU, S.H., et al., "Low shrinkage light curable nanocomposite for dental restorative material", Dental Materials, v. 22, n. 1, pp. 138-145, 2006.

[10] FONSECA, A.S., Odontologia estética: a arte da perfeição, 1ed., São Paulo, Artes Médicas, 2008.

[11] RODRIGUES, R.A., REBELATTO, C., BASTOS, R.A., et al., "Análise de microdureza Knoop de 4 tipos de resinas compostas através do microdurômetro digital HVS-1000", Odontologia Clínico Científica, v. 9, n. 3, pp. 55-58, Jan.-Mar. 2010.

[12] MONTENEGRO R.V., ARRAIS, G.L.R., FRANÇA, K.P., et al. "Efeito da partícula de carga e do tempo de armazenamento sobre a microdureza de superfície de compósitos restauradores estéticos", Pesquisa Brasileira Odontopediátrica Clínica Integrada, v. 10, n. 3, pp. 345-350, Set.-Dez. 2010.

[13] BEUN, S., GLORIEUX, T, DEVAUX, J., et al., "Characterization of nanofiled compared to universal and microfilled composites", Dental Materials, v. 23, n. 1, pp. 51-59, 2007.

[14] KIM, K.H., ONG, J.L., OKUNO, O., "The effect of filler loading and morphology on the mechanical properties of contemporary composites", The Journal of Prosthetic Dentistry, v. 87, n. 6, pp. 6432-649, Jun. 2002.

[15] MONDELLI, J. Restaurações Estéticas. São Paulo, Sarvier, 1984.

[16] AW, T.C., NICHOLLS, J.I., "Polymerization shrinkage of densely-filed resin composite", Operative Dentstry, v. 16, n. 5, pp. 498-504, Set.-Out. 2001.

[17] MUNKSGAARD, E.C., HANSEN, E.K., KATO, W., "Wall-to-wall polymerization contraction of composite resins versus filler content", Scand J Dent Res, v. 87, pp. 526-531, 1987.

[18] TANIMOTO, Y., KITAGAWA, T., AIDA, M., et al., "Experimental and computational approach for evaluating the mechanical characteristics of dental composite resins with various filler sizes", Acta Biomaterialia, v. 2, n. 6, pp. 633-639, Set. 2006.

[19] MIYASAKA, T., "Effect of shape and size silanated fillers on mechanical properties of experimental photo cure composite resins", Dental Materials, v. 15, n. 2, pp. 98-110, 1996.

[20] PUCKETT, A.D., FITCHIE, J.G, KIRK, P.C, et al., "Direct composite restorative materials", The Dental Clinics of North America, v. 51, n. 1, pp. 659-675, 2007. 